\title{
Evaluation of Prescribing and Administering As-Needed Pain Medications Based on Pain Severity Scores
}

\author{
Jenna Stearns, PharmD; Christine Cortese, PharmD, BCPS; Jennifer Remington, PharmD; Nirav Patil, MBBS, MPH
}

University Hospitals Richmond Medical Center

\begin{abstract}
Pain is a common symptom reported by patients admitted to hospitals in both medical and surgical units. Due to the subjective and multidimensional nature of pain, it should be assessed regularly to ensure patient pain control. Suboptimal prescribing of opioids and other pain medications contribute to the inadequate treatment of pain. To combat the wide variability and response to pain medications, many providers prescribe as-needed range orders of pain medications to cover each pain severity. These range orders enable necessary and safe adjustments in dose based on individual responses to treatment. Currently, there are no studies analyzing the prescribing of pain medications based on pain severity, leaving a gap in literature in inpatient pain management. The purpose of this retrospective cohort study was to evaluate the appropriateness of prescribing as-needed pain medications based on the patient's pain severity scores throughout their hospital stay at a 125-bed community hospital in Northeast Ohio on the general medicine floor. Secondarily, this study also evaluated the administration of pain medications by nursing staff based on patient-reported pain severity scores. Statistical analyses including Chi-square tests, $t$-tests, Fisher's Exact tests and descriptive statistics were utilized to determine the significance of the data collected. This study found that there was a statistically significant difference between appropriately (47.4\%) and inappropriately (52.6\%) prescribed pain medications ( $P<0.001)$. There was also a statistically significant difference between appropriately (40.5\%) and inappropriately (59.5\%) administered pain medications $(P<0.001)$. Pharmacists hope to improve pain management practices by providing education to both providers and nurses to prevent poor patient outcomes and uncontrolled pain.
\end{abstract}

Keywords: Pain management; Pain assessment; Prescribing; Administering; Inpatient; Opioids

\section{Introduction}

Pain is a common symptom reported by patients admitted to hospitals in both medical and surgical units., ${ }^{1,2}$ Due to the subjective and multidimensional nature of pain, it should be assessed regularly to ensure patient pain control. To combat the wide variability and response to pain medications, many providers prescribe as-needed range orders of pain medications to cover each pain severity. According to the American Society for Pain Management Nursing and the American Pain Society, range orders "enable necessary and safe adjustments in dose based on individual responses to treatment."3

There are many factors to consider when prescribing pain medications for patients admitted to the hospital, including (but not limited to) patient age, type and intensity of pain, duration of pain, past and prior response to analgesia, comorbidities, and end-organ function. ${ }^{1-4}$ Insufficient prescribing of opioids and other pain medications contribute to the inadequate treatment of pain. ${ }^{1-4}$

Corresponding author: Jenna Stearns, PharmD

University Hospitals Richmond Medical Center

27100 Chardon Road

Richmond Heights, OH 44143

Phone: 440-585-1319

Email: jenna.stearns@uhhospitals.org
The Joint Commission requires hospital health systems to provide staff with educational resources and programs "to improve pain assessment, pain management and the safe use of opioid medications based on the identified needs of its patient population." ${ }^{5}$ Part of their requirement is ensuring patients have pain management coverage for each pain severity score. ${ }^{5}$ One way to assess that the prescribed pain medications will cover each pain severity score is to evaluate if the orders have increasing morphine equivalent doses as the severity increases. For example, a moderate pain medication order should have a lower morphine equivalent dose compared to a severe pain medication order.

In a retrospective and prospective study by Lin et al., the authors aimed to "identify risk factors for the undertreatment of acute pain and system-related, institution-specific barriers to high-quality acute pain management through survey questionnaires and patient interviews." 6 This study evaluated patient satisfaction with pain management and the authors found that the "most common system-related barrier perceived by patients is delay in patient interventions." ${ }^{\prime 6}$

Additionally, a retrospective cohort study by Wei et al., the authors identified the "association of pain severity with resource utilization and costs and characterized the association of opioid use while controlling for pain severity." ${ }^{17}$ They found that "increased pain severity and opioid use were independently associated with higher resource utilization and cost." ${ }^{\prime 7}$ With better prescribing and administration of pain 
medications, our health system could improve patient care while also improving resource utilization.

While there is literature to support the patient pain management experience as well as cost utilization, there are no studies that analyze the prescribing of pain medications based on pain severity, leaving a gap in the literature with respect to inpatient pain management. The purpose of this study was to evaluate the prescribing of as-needed pain medications based on the patient-reported pain severity scores throughout their hospital stay on the general medicine floor of University Hospitals Richmond Medical Center (UHRMC), a 125-bed community hospital located in northeast Ohio. Secondarily, this study will also evaluate the administration of these pain medications by nursing staff based on patient-reported pain severity scores. This study sought to identify areas of improvement for prescribing and administering inpatient pain medications as well as find areas for pharmacist intervention in pain management.

\section{Methods}

Instrumentation and Implementation

The protocol for this study was sent to and approved by the University Hospitals Health System Institutional Review Board (IRB) in December of 2020. After IRB approval, data was obtained by thorough chart review of the University Hospitals electronic medical record UHCare through Allscripts ${ }^{\circledR}$.

\section{Study Design}

This retrospective cohort evaluated the appropriateness of prescribing pain medication of attending physicians, medical residents and nurse practitioners on the general medicine floor of UHRMC. It also evaluated the administration of pain medication amongst nurses based on patient-reported pain scores as a secondary endpoint.

Patients were identified by the primary author through extraction of records using the hospital's electronic medical record from January 1, 2020 through June 30, 2020 of all pain medications prescribed and administered on the general medicine floor. The medications used for data extraction were the pain medications included in the admission and pain order sets, as they are the most common medications used to treat pain while inpatient. Orders were eliminated from data collection if the patient was receiving the medication scheduled (not as-needed). The following medications were included: oxycodone, oxycodone/acetaminophen, tramadol, morphine, hydromorphone, codeine/acetaminophen, hydrocodone/ acetaminophen, acetaminophen, ibuprofen, and ketorolac.

To be included, patients had to be 18 years of age or older, be admitted to UHRMC general medicine floors, and have a pain medication prescribed for each pain severity (mild pain, moderate pain, and severe pain). Exclusion criteria included patients that were under the age of 18 , without pain medications indicated for each pain severity, with a cancer or sickle cell diagnosis, admitted to the Intensive Care Unit (ICU), under hospice care, or not admitted to UHRMC (ie. discharged from the emergency department or transferred to another hospital in the health system).

"Appropriate prescribing" in this study was defined as pain medications that were ordered with increasing morphine equivalent dose proportionate to the severity of pain. For example, an order indicated for moderate pain severity should have a lower morphine equivalent dose than an order for severe pain and a higher morphine equivalent dose than the mild pain order. Alternatively, "inappropriate prescribing" was defined as pain medications ordered with morphine equivalent doses that were disproportionate to the patient's pain severity. For example, if the moderate pain severity medication had a higher morphine equivalent dose than the severe pain medication that would be considered inappropriate. "Appropriate administration" was defined as a pain medication that was administered according to the prescriber's order and the patient's reported and documented pain severity score. "Inappropriate administration" was defined as a pain medication that was given for a pain score outside of the prescriber's order or without a documented pain score. For example, if a patient reported a pain score in the moderate category and was given a medication indicated for mild or severe pain, this would constitute inappropriate administration.

With a population size of 200 patients, in order to have a $95 \%$ confidence interval with $5 \%$ margin of error, this study required at least 132 patients to meet power. Statistical analysis was completed by the statistician utilizing SAS Enterprise Guide, version 7.1.

\section{Statistical Analysis}

Descriptive statistics, including mean and median, were used to analyze the demographic data. Chi-square tests were utilized to determine medication order appropriateness, and T-tests were utilized to compare the percentage differences between appropriately or inappropriately ordered pain medications for each pain severity. The statistical test utilized to compare appropriately and inappropriately administered medications was Fisher's Exact test.

\section{Results}

\section{Demographic Characteristics}

There were 143 patients who met the study's inclusion criteria and were therefore eligible for statistical analysis. Demographic characteristics are represented by Table 1 . The average age of the study population was 60.5 years old. In total, $39.9 \%$ were male and $60.1 \%$ were female. The average length of stay was about 3 days. Of the pain management medications ordered, $16.1 \%$ were entered by an attending physician, $17.5 \%$ entered by a nurse practitioner, and $66.4 \%$ by a resident physician. 
Study Endpoints

The primary endpoint of this study was the percentage of appropriately ordered pain regimens for each pain severity score. With the 143 included patients, there were 529 orders for pain medications. Of the 529 orders, $47.4 \%$ were ordered appropriately with $52.6 \%$ ordered inappropriately $(p<0.001)$ [Figure 1]. When comparing the appropriateness of prescribing pain medications between pain severity categories (mild, moderate and severe pain), $88.1 \%$ of mild pain medications, $34.3 \%$ of moderate pain medications and $28.6 \%$ of severe pain medications were ordered appropriately $(P<0.001)$ [Figure 2]. The bulk of the inappropriately ordered pain medications were in the moderate and severe categories making up $65.7 \%$ and $71.4 \%$, respectively $(P<0.001)$.

The secondary endpoint was the percentage of appropriately administered pain medications based on the patient-reported and documented pain severity scores. Of the $\mathbf{5 2 9}$ orders for pain medications, there were 393 orders where at least one dose was given. There were 234 orders (59.5\%) that had at least one dose of a medication given inappropriately $(P<0.001)$ [Figure 3]. Of those 234 orders, there were 75 instances where a dose of a medication was given for a higher reported pain score (ie. a moderate severity pain medication was given for a severe pain score), 44 instances where a dose was given for a lower reported pain score (ie. a severe pain severity medication was given for a moderate or mild pain score), and 178 instances where a medication was given without a documented pain score $(P<0.001)$ [Figure 4]. These values exceed the 234 orders due to the fact that one medication order often allowed for multiple medication administrations, with some of the administration occurring outside of the provider's order. For example, if tramadol was ordered for moderate pain and five doses were given, there were instances where it was given for a high pain score and a lower pain score than indicated.

\section{Discussion}

Pain management is an important part of patient care in all healthcare settings. Poor prescribing of opioids and other pain medications contributes to the inadequate treatment of pain. ${ }^{1-}$

${ }^{4}$ It is important to have a pain medication ordered to cover each pain severity score with increasing morphine-equivalent doses as the severity increases (i.e. a moderate pain medication order should have a lower morphine equivalent dose compared to the severe pain medication order).

The primary endpoint of this study was to evaluate the prescribing of as-needed pain medications based on patientreported pain severity scores throughout their hospital stay. There was a statistically significant difference between orders that were prescribed appropriately and those that were prescribed inappropriately. Less than half of the pain management orders (47.4\%) were prescribed appropriately. Mild pain scores were much more likely to be treated appropriately when compared with moderate and severe pain scores (with severe being the least likely). One could speculate that the types of medications used for moderate or severe pain (which were most often opioids) may contribute to this.

The secondary endpoint of this study was to evaluate the administration of as-needed pain medications based on patient-reported pain severity scores throughout their hospital stay. There was a statistically significant difference between orders that were administered appropriately and those that were administered inappropriately. Over half of the orders administered had at least one instance where a dose was given incorrectly (59.5\%). There were 75 orders where either a moderate or mild pain severity medication was given for a higher pain score where a higher pain severity medication was indicated. There were 44 orders where a medication for severe or moderate pain was given for a lower pain score, and there were 178 orders where a pain medication was given without a documented pain score.

\section{Strengths and Limitations}

The population size included was enough for this study to be adequately powered. There were also statistically significant differences between all of the study endpoints which identified areas of improvement for both prescribing and administering pain medications. The selected timeframe also limited prescribing variability by only including one class of medical residents. This study will also contribute to the limited body of literature available for prescribing and administering pain medications to improve inpatient pain management.

It should be noted that there are limitations to this study. This study had a limited time frame of six months, was singlecentered and retrospective. Additionally, the single-centered nature of this study may limit the generalizability of the results. The secondary endpoint of nursing administration relied on timely and accurate documentation of pain scores. For example, a medication could have been given appropriately, but if the nurse never documented the score, the medication was reported as administered with no pain score. The data only analyzed errors specific to administration instead of prescribing, which could be an area for future research.

\section{Future Direction and Clinical Practice}

While this study provided valuable information regarding the prescribing and administration of pain medications for UHRMC, there are additional endpoints and areas of study that could be valuable in improving inpatient pain management. An additional endpoint for this study would be to evaluate specific prescribing errors to better improve prescribing practices moving forward. Another would be to include other hospitals in the health system and lengthen the study timeframe to provide larger scale data with more generalizable results.

Pharmacists are an asset in pain management and would help improve current prescribing and administration at UHRMC by providing medication recommendations, ensuring Joint Commission requirements are met, and providing medication 
counseling to patients and families. Pharmacists would also be valuable in providing education to providers regarding morphine equivalents, especially between the different dosage forms available (parenteral, oral, patch, etc.). Lastly, pharmacists could also provide nursing education on patient pain assessment as well as documentation of pain scores to ensure medications are given for the appropriate pain score.

Acknowledgements: The author would like to acknowledge Christine Cortese and Jennifer Remington for their advising on this project as well as Nirav Patil, the statistician, who was able to assist with analyzing the results.

Conflicts of Interest: We declare no conflicts of interest or financial interests that the authors or members of their immediate families have in any product or service discussed in the manuscript, including grants, employment, gifts, stock holdings or options, honoraria, consultancies, expert testimony, patents and royalties.

Treatment of Human Subjects: IRB exemption granted

\section{References}

1. Damico V, Murano L, Cazzaniga F, Dal Molin A. Pain prevalence, severity, assessment and management in hospitalized adult patients: a result of a multicenter cross sectional study. Ann 1st Super Sanita. 2018;54(3):194-200.

2. Murnion BP, Gnjidic D, Hilmer SN. Prescription and administration of opioids to hospital in-patients, and barriers to effective use. Pain Medicine. 2010 Jan;11(1):5866.

3. Drew DJ, Gordon DB, Morgan B, Manworren RCB. "Asneeded" range orders for opioid analgesics in the management of pain: a consensus statement of the American Society for Pain Management Nursing and the American Pain Society. Pain Management Nursing. 2018 June;19(3):207-210.

4. Dix P Sandhar B Murdoch J Maclntyre PA. Pain on medical wards in a district general hospital. Br J Anaesth 2004;92:235-7

5. The Joint Commission. Pain assessment and management standards for hospitals. R3 Report (Requirement, Rationale, Reference). 2017 Aug 29;11:1-7.

6. Lin RJ, Reid MC, Liu LL, et al. The barriers to high-quality patient management: a qualitative study. Am J Hosp Palliat Care. 2015 September;32(6):594-599.

7. Wei W, Gandhi K, Blauer-Peterson C, et al. Impact of pain severity and opioid use on health care resource utilization and costs among patients with knee and hip osteoarthritis. Journal of Managed Care and Specialty Pharmacy. 2019 Sept;25(9):957-965. 
Table 1. Demographic Characteristics

\begin{tabular}{|c|c|}
\hline & $\mathbf{N}=\mathbf{1 4 3}$ \\
\hline Age & \\
Mean \pm SD & $60.5 \pm 17.6$ \\
& $62(49,72)$ \\
Median (25 ${ }^{\text {th }}$ and $75^{\text {th }}$ percentile) & \\
\hline Sex, n (\%) & $57(39.9 \%)$ \\
Male & $86(60.1 \%)$ \\
Female & $3(2,5)$ \\
\hline Length of Stay & \\
\hline Provider Entered & $95(66.4 \%)$ \\
Resident & $25(17.5 \%)$ \\
NP & $23(16.1 \%)$ \\
Attending & \\
\hline
\end{tabular}

Figure 1. Appropriateness of prescribing pain medications

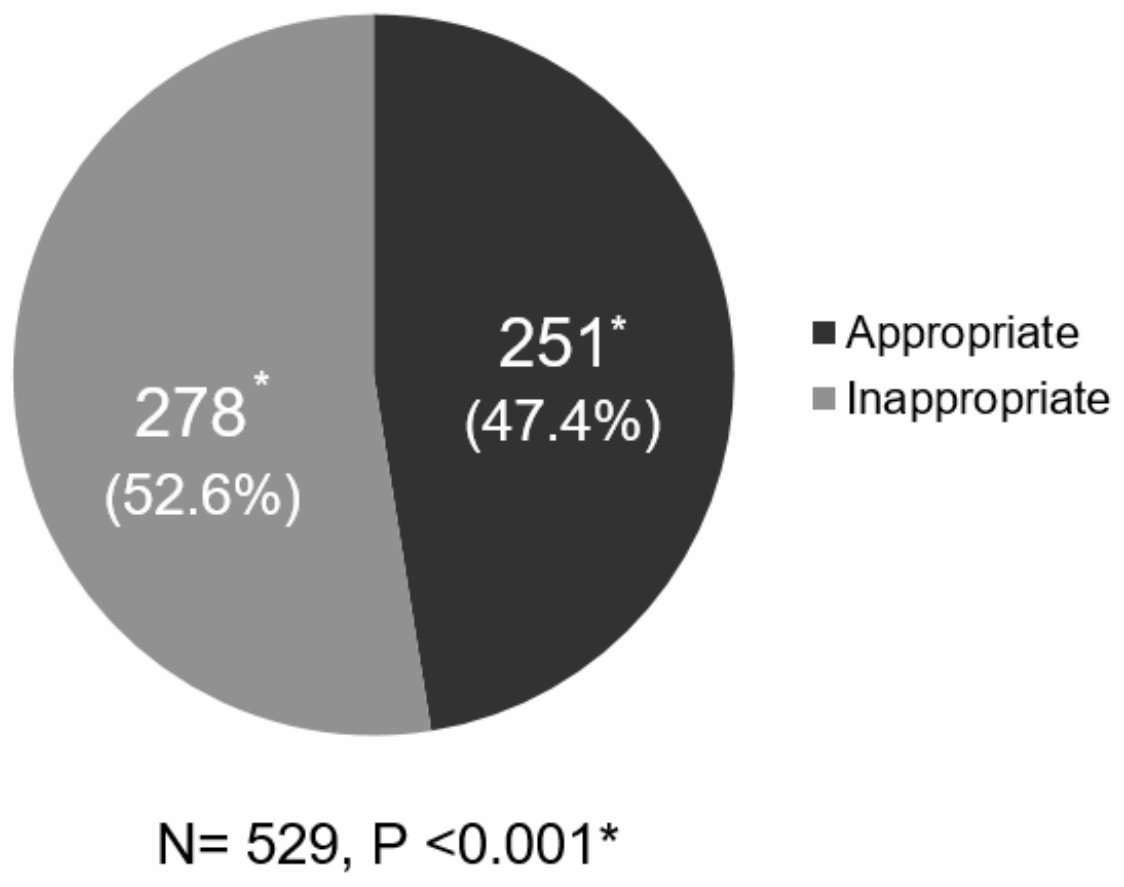


Figure 2. Appropriateness of prescribing pain medications for each pain severity

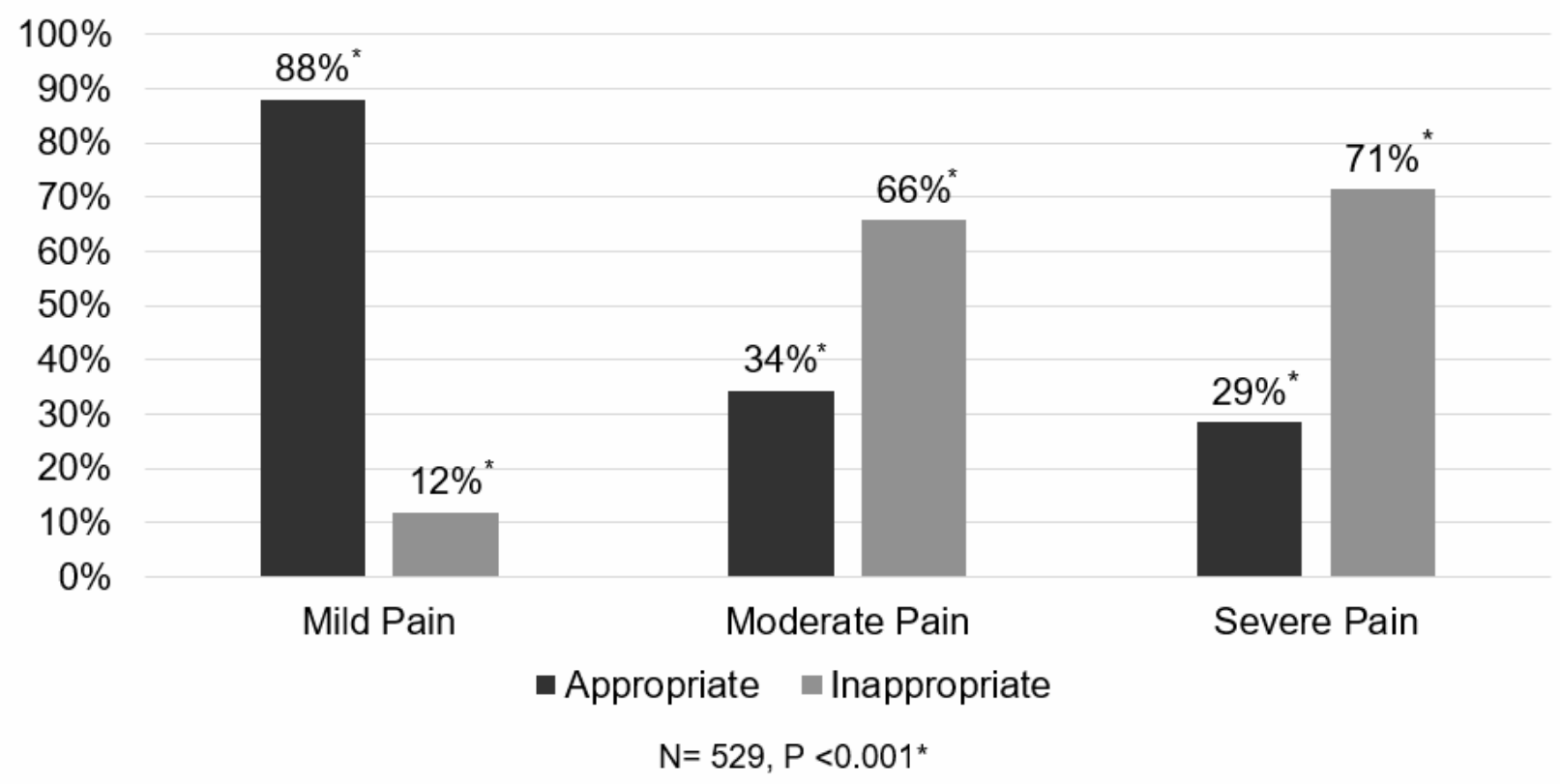

Figure 3. Appropriateness of administering pain medications based on pain severity scores

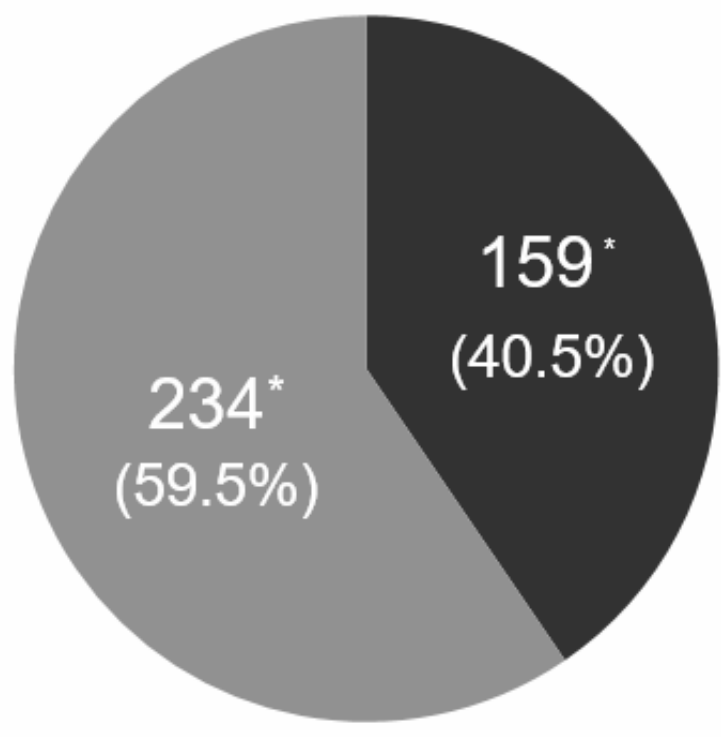

- Appropriate

- Inappropriate

$$
N=393, P<0.001^{*}
$$


Figure 4. Inappropriately administered pain medications

200

180

160

140

120

100

80

\section{$75^{*}$}

60

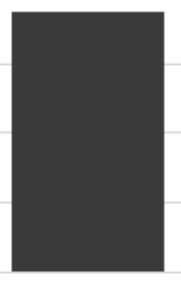

$44^{*}$

40

20

0

Higher Pain Score

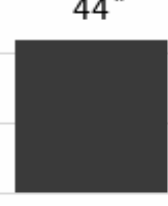

Lower Pain Score

- Instances

$N=234, P<0.001^{*}$
$178^{*}$

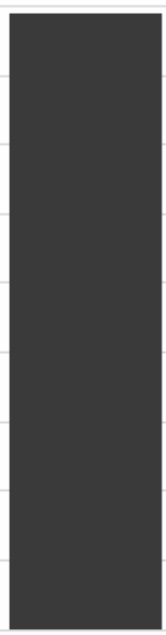

No Pain Score 